PROOF COPY 010607JAS

\title{
A stable boundary element method for modeling transient acoustic radiation
}

\author{
D. J. Chappell, a) P. J. Harris, D. Henwood, and R. Chakrabarti \\ School of Computing and Mathematical Sciences, University of Brighton, Lewes Road, \\ Brighton BN2 4GJ, United Kingdom
}

(Received 10 October 2005; revised 13 April 2006; accepted 17 April 2006)

\begin{abstract}
Transient acoustic radiation from a closed axisymmetric three-dimensional object is modeled using the time domain boundary element method. The widely reported instability problems are overcome by reformulating the integral equation to obtain a Burton and Miller type equation in the time domain. The stability of such an approach is mathematically justified and supported by subsequent numerical results. The hypersingular integrals which arise are evaluated using a method valid for any surface discretization. Numerical results for the radiation of a spherical wave are presented and compared with an exact solution. The accuracy and stability of the results are verified for several geometrically different radiating objects. (C) 2006 Acoustical Society of America.
\end{abstract}

[DOI: 10.1121/1.2202909]

PACS number(s): 43.20.Px, 43.20.Bi [SFW]

Pages: 1-XXXX

\section{INTRODUCTION}

Time domain boundary integral methods have been used to solve wave propagation problems since the $1960 \mathrm{~s} .{ }^{1,2}$ Since then increasing computer power has made numerical solutions possible over longer run times and so long-time instabilities in the time marching numerical solutions have become evident. ${ }^{3-5}$ A number of methods have been suggested to resolve this such as time-averaging ${ }^{6,7}$ and modified1 time-stepping. ${ }^{4}$ Using an implicit formulation with high order interpolation and quadrature was also found to give stable results for all practical purposes. ${ }^{8,9}$ Ha-Duong et al. ${ }^{10}$ obtained stable results using a Galerkin approach and used an energy identity to prove stability of the Galerkin approximation. However, Galerkin methods are difficult and costly to implement and so have remained relatively unpopular despite their theoretical advantages.

The cause of these instabilities is discussed in Ref. 6 (in the context of the electric field integral equation) and is shown to be related to internal resonances of the scattering (or radiating) body. A similar argument is applied to the methods for acoustics problems later in this paper. In order to prevent these instabilities a time domain Burton-Miller type integral equation formulation like that originally applied to frequency domain problems ${ }^{11}$ is used. A time domain formulation was proposed by Michielssen et al. ${ }^{12}$ for acoustics scattering problems. It is shown that this formulation avoids the solution being corrupted by internal resonances and thus allows stability. The main difficulty in applying this method is evaluating the hypersingular integrals which are introduced. One method for doing this is to apply a similar limiting procedure to that of Terai ${ }^{13}$ in the frequency domain as in Refs. 12 and 14. However, this has the disadvantage of being restricted to piecewise flat surface discretizations. The method employed here reformulates the hypersingular inte-

${ }^{a)}$ Electronic mail: d.j.chappell@brighton.ac.uk grals into weakly singular ones using a Taylor series expansion and the identity of Meyer et al. $^{15}$

\section{THE INTEGRAL EQUATION FORMULATION}

Let $\Omega \subset R^{3}$ be a finite object with regular boundary surface $\Gamma$. Let $\Omega_{+}=\mathbb{R}^{3} \backslash \bar{Q}$ denote the unbounded exterior acoustic field and $\Omega_{-}=\Omega \backslash \Gamma$ denote the interior of $\Omega$. Assume that $\Omega_{+}$is filled with a homogeneous compressible acoustic medium with speed of sound $c$. Let the radiated velocity potential be denoted by $\varphi:\left(\Omega_{+} \cup \Gamma\right) \times \mathbb{R}_{\geqslant 0} \rightarrow \mathbb{R}$ and consider the following initial-boundary value problem:

$$
\begin{aligned}
& \nabla^{2} \varphi(\underline{x}, t)=\frac{1}{c^{2}} \frac{\partial^{2} \varphi}{\partial t^{2}}(\underline{x}, t) \quad \text { in }\left(\Omega_{+} \cup \Gamma\right) \times \mathbb{R}_{\geqslant 0}, \\
& \varphi(\underline{x}, 0)=0 \quad \text { in } \Omega_{+} \cup \Gamma, \\
& \dot{\varphi}(\underline{x}, 0)=0 \quad \text { in } \Omega_{+} \cup \Gamma, \\
& \frac{\partial \varphi}{\partial \underline{\hat{n}}_{x}}(\underline{x}, t)=f(\underline{x}, t) \quad \text { on } \Gamma \times \mathbb{R}_{\geqslant 0},
\end{aligned}
$$

where $\hat{n}_{x}$ denotes the outward unit normal vector to $\Gamma$ at $\underline{x}, \dot{\varphi}$ the time derivative and $f: \Gamma \times \mathbb{R}_{\geqslant 0} \rightarrow \mathrm{R}$ is known. It is well known that this is a well-posed problem (see, for example, Ref. 10).

The initial-boundary value problem above may be represented by the following integral equation using Green's second identity ${ }^{16}$

$$
\mathcal{D}(\varphi(\underline{x}, t))-\mathcal{S}\left(\frac{\partial \varphi}{\partial \underline{\hat{n}}_{x}}(\underline{x}, t)\right)=\left\{\begin{array}{cc}
\frac{1}{2} \varphi(\underline{x}, t) & \underline{x} \in \Gamma \\
\varphi(\underline{x}, t) & \underline{x} \in \Omega_{+},
\end{array}\right.
$$

where in the case $\underline{x} \in \Gamma$, it is assumed that $\Gamma$ is locally differentiable at $\underline{x}$. The single-layer potential $\mathcal{S}$, and the doublelayer potential $\mathcal{D}$ are defined as usual in terms of the fundamental solution of the wave equation 


\section{PROOF COPY 010607JAS}

$$
G(\underline{x}, t):=\frac{1}{4 \pi|\underline{x}|} \delta\left(t-\frac{|\underline{x}|}{c}\right),
$$

where $\delta$ is the Dirac delta function. Explicitly, they are defined by

$$
\mathcal{S} \varphi(\underline{x}, t):=\int_{0}^{t^{+}} \int_{\Gamma} G(\underline{x}-\underline{\xi}, t-s) \varphi(\underline{\xi}, s) d S_{\xi} d s
$$

and

$$
\mathcal{D} \varphi(\underline{x}, t):=\int_{0}^{t^{+}} \int_{\Gamma} \frac{\partial G}{\partial \underline{\hat{n}}_{\xi}}(\underline{x}-\underline{\xi}, t-s) \varphi(\underline{\xi}, s) d S_{\xi} d s,
$$

where $t^{+}=t+\varepsilon$ for arbitrarily small $\varepsilon$. This avoids taking the upper limit on the time integral at the singularity in $\delta$. The classical jump relations in the form $[\mathcal{S} \varphi]=0$ and $[\mathcal{D} \varphi]=\varphi$ hold, where [-] represents the jump across $\Gamma$. It is easy to show that the integral equation (5) is equivalent to the wellknown Kirchoff or retarded potential integral equation: ${ }^{17}$

$$
\begin{aligned}
& \frac{-1}{4 \pi} \int_{\Gamma}\left\{\frac{\partial R}{\partial \hat{n}_{\xi}}\left(\frac{\varphi(\underline{\xi}, \tau)}{R^{2}}+\frac{\dot{\varphi}(\underline{\xi}, \tau)}{c R}\right)+\frac{1}{R} \frac{\partial \varphi}{\partial \underline{\hat{n}}_{\xi}}(\underline{\xi}, \tau)\right\} d S_{\xi} \\
& \quad= \begin{cases}\frac{1}{2} \varphi(\underline{x}, t) \quad \underline{x} \in \Gamma \\
\varphi(\underline{x}, t) \quad \underline{x} \in \Omega_{+} .\end{cases}
\end{aligned}
$$

Here $R=|\underline{x}-\underline{\xi}|$ and $\tau=t-R / c$ is the retarded time.

\section{A. Stability problems}

As mentioned previously, it is well known that time marching solutions obtained using Eq. (6) suffer from poor stability. Since $\partial \varphi / \partial \hat{n}_{\xi}$ is known for all time with $\underline{x} \in \Gamma$, the term containing it will not affect the stability of the time marching solution. To study the stability properties it is therefore sufficient to consider the case where this term is zero, hence Eq. (6) becomes

$$
\frac{1}{2} \varphi(\underline{x}, t)=\frac{-1}{4 \pi} \int_{\Gamma} \frac{\partial R}{\partial \underline{\hat{n}}_{\xi}}\left(\frac{\varphi(\underline{\xi}, \tau)}{R^{2}}+\frac{\dot{\varphi}(\underline{\xi}, \tau)}{c R}\right) d S_{\xi} .
$$

The initial conditions mean the only time harmonic solutions that should be admitted by Eq. (7) are zero. However, supposing that $\varphi(\underline{x}, t)=\hat{\varphi}(\underline{x}) e^{-i \omega t}$ with $\omega \in \mathbb{R}_{\geqslant 0}$ is a time harmonic solution of Eq. (7) valid for $t \geqslant t^{\prime}>0$, where $t^{\prime}$ is some arbitrary positive time gives

$$
\frac{1}{2} \hat{\varphi}(\underline{x}) e^{-i \omega t}=\frac{-1}{4 \pi} \int_{\Gamma} \frac{\partial R}{\partial \underline{\hat{n}}_{\xi}} \hat{\varphi}(\underline{\xi}) e^{-i \omega t} \frac{e^{i k R}(1-i k R)}{R^{2}} d S_{\xi},
$$

where $k:=\omega / c$ is the wave number. Hence the following equation for $\hat{\varphi}$ is obtained

$$
\frac{1}{2} \hat{\varphi}(\underline{x})=\int_{\Gamma} \frac{\partial}{\partial \underline{\hat{n}}_{\xi}}\left(\frac{e^{i k R}}{4 \pi R}\right) \hat{\varphi}(\underline{\xi}) d S_{\xi},
$$

which is the well-known analogue of Eq. (7) in the frequency domain. This equation is known to admit nontrivial solutions for a set of discrete resonant wave numbers. ${ }^{11}$ Hence Eq. (7) must also admit these resonant solutions for $t \geqslant t^{\prime}>0$.
The fact that these resonant time harmonic solutions are admitted by Eq. (7) is not in itself a cause of instability. However, the solution of the discretized version of Eq. (7) may be considered as a sum of discrete frequency components of the form $\hat{\varphi}(\underline{x}) e^{\nu t}$ with $\nu \in \mathrm{C}$. Time harmonic solutions occur here when the sum is over a single value of $\nu$ lying on the imaginary axis. As in the case of the electric field integral equation studied in Ref. 6, the discretization leads to a loss of accuracy causing the values of $\nu$ to deviate from their theoretical values. This loss in accuracy is more severe for higher frequencies. For a time discretization with time-step $\Delta t$, angular frequencies $\omega \geqslant \pi / \Delta t$ cannot be represented by the discrete model (Nyquist condition) and for $\omega$ $\approx \pi / \Delta t$ the discrete model will be highly inaccurate and hence so will the values of $\nu$ in the calculation. ${ }^{6}$ This is a problem since the resonances become more dense as $\omega$ increase ${ }^{11}$ and so the smaller $\Delta t$ is taken to be (necessary to approximate higher frequency radiation), the greater the chance that $\omega_{\text {res }} \approx \pi / \Delta t$ for some resonant angular frequency $\omega_{\text {res }}$. If this occurs, then the value of $\nu$ corresponding to the resonance will be calculated inaccurately and may in general move off the imaginary axis into the right or left half planes. Any excited resonances for which $\nu$ moves into the right half plane give rise to an exponentially increasing oscillation or instability.

\section{B. Burton-Miller-type time domain integral equation}

In order to cure the above-described instability problems, a time domain analogue of the well-known Burton and Miller method in the frequency domain is used to obtain a solution for $\varphi$ on $\Gamma$. A similar formulation to the one suggested in Ref. 12 is adapted to apply to radiation problems. This entails taking a linear combination of the derivative of Eq. (5) with respect to time and the normal derivative of Eq. (5). Explicitly this yields

$$
\begin{aligned}
(1- & \alpha)\left\{\mathcal{D}(\dot{\varphi}(\underline{x}, t))-\mathcal{S}\left(\left(\frac{\dot{\partial} \varphi}{\partial \underline{\hat{n}}_{x}}\right)(\underline{x}, t)\right)-\frac{1}{2} \dot{\varphi}(\underline{x}, t)\right\} \\
& +\alpha c\left\{\frac{\partial}{\partial \hat{\hat{n}}_{x}} \mathcal{D}(\varphi(\underline{x}, t))-\frac{\partial}{\partial \underline{\hat{n}}_{x}} \mathcal{S}\left(\frac{\partial \varphi}{\partial \underline{\hat{n}}_{x}}(\underline{x}, t)\right)-\frac{1}{2} \frac{\partial \varphi}{\partial \underline{\hat{n}}_{x}}(\underline{x}, t)\right\} \\
& =0
\end{aligned}
$$

where $\alpha \in(0,1)$ is a coupling parameter. The factor of $c$ is required to balance the relative sizes of the terms in the two equations, i.e., so the power of $c$ in the coefficients of like terms is the same in both equations. Note that the following easily proven identity has been used here:

$$
\frac{\partial}{\partial t}(\mathcal{S} \text { or } \mathcal{D}) \varphi=(\mathcal{S} \text { or } \mathcal{D}) \dot{\varphi} .
$$

Equation (10) may also be written in a similar form to Eq. (6). The upper (time derivative) term is like Eq. (6) for $\underline{x}$ $\in \Gamma$, but with $\dot{\varphi}$ substituted for $\varphi$ and the right-hand side subtracted. The lower (normal derivative) term is more complicated and contains hypersingular integrals.

The methods used to evaluate these will be discussed later. Note that in this integral equation formulation the val- 
ues of $(\dot{\partial} \varphi / \partial \hat{n})$ on $\Gamma \times \mathbb{R}_{\geqslant 0}$ are also required. However, the same initial-boundary value problem $(1,2,3,4)$ is being solved and so these values may be computed from Eq. (4), assuming $f$ is differentiable with respect to time. In the case where $f$ is given by a closed expression this may be done directly by differentiating, as is done in the examples considered later. In the case where $f$ is obtained from discrete measured values such as in loudspeaker modeling, the easiest way to calculate $\dot{f}$ is by using a finite difference approximation.

The use of the Burton-Miller type integral equation (10) to avoid the above-described stability problems is now considered in detail. It is clear from the previous section that the stability problems will be avoided if the integral equation arising when homogeneous boundary conditions are applied does not admit nontrivial solutions. The Burton-Miller-type equation with homogeneous boundary conditions is given by

$$
(1-\alpha)\left\{\mathcal{D}(\dot{\varphi}(\underline{x}, t))-\frac{1}{2} \dot{\varphi}(\underline{x}, t)\right\}+\alpha c\left\{\frac{\partial}{\partial \hat{n}_{x}} \mathcal{D}(\varphi(\underline{x}, t))\right\}=0 .
$$

Assume that $\varphi$ is a nontrivial solution of Eq. (12) and consider the double-layer potential

$$
U(\underline{x}, t)=\mathcal{D} \varphi(\underline{x}, t)
$$

for any $\underline{x} \in \mathbb{R}^{3}$. The jump relations together with Eqs. (11) and (12) give the following relation between interior boundary values of $\dot{U}$ and $\partial U / \partial \underline{\hat{n}}_{x}$ :

$$
(1-\alpha) \dot{U}_{-}+\alpha c\left(\frac{\partial U}{\partial \underline{\hat{n}}_{x}}\right)_{-}=0
$$

where the subscript "-" denotes the interior boundary values. Applying Green's first identity to $U$ and $\dot{U}$ on $\Omega_{-}$gives

$$
\begin{gathered}
\frac{1}{2} \int_{\Omega_{-}}\left\{\left|\nabla_{\xi} U\left(\underline{x}, t^{+}\right)\right|^{2}+\frac{\left|\dot{U}\left(\underline{x}, t^{+}\right)\right|^{2}}{c^{2}}\right\} d V_{\xi} \\
=\int_{0}^{t^{+}} \int_{\Gamma} \dot{U}_{-}\left(\frac{\partial U}{\partial \underline{\hat{n}}_{x}}\right) d S_{\xi} d s,
\end{gathered}
$$

assuming $\varphi$ is such that the volume integral is always finite. Substituting Eq. (13) into Eq. (14) and noting that $\alpha$ $\in(0,1)$ yields

$$
\begin{aligned}
0 & \leqslant \frac{1}{2} \int_{\Omega_{-}}\left\{\left|\nabla_{\xi} U\left(\underline{x}, t^{+}\right)\right|^{2}+\frac{\left|\dot{U}\left(\underline{x}, t^{+}\right)\right|^{2}}{c^{2}}\right\} d V_{\xi} \\
& =\frac{-\alpha c}{(1-\alpha)} \int_{0}^{t^{+}} \int_{\Gamma}\left|\frac{\partial U}{\partial \hat{n}_{x}}\right|_{-}^{2} d S_{\xi} d s \\
& =\frac{-(1-\alpha)}{\alpha c} \int_{0}^{t^{+}} \int_{\Gamma}|\dot{U}|_{-}^{2} d S_{\xi} d s \leqslant 0 .
\end{aligned}
$$

This means that both $\dot{U}_{-}$and $\left(\partial U / \partial \hat{n}_{x}\right)_{-}$are zero. The jump relations give that

$$
\dot{U}_{+}=\dot{U}_{-}+\dot{\varphi}=\dot{\varphi}, \quad\left(\frac{\partial U}{\partial \underline{\hat{n}}_{x}}\right)_{+}=\left(\frac{\partial U}{\partial \underline{\hat{n}}_{x}}\right)_{-}=0
$$

where the subscript "+" denotes the exterior boundary values. This means that $U$ satisfies the homogeneous Neumann boundary condition. By uniqueness of the exterior Neumann problem $U=0$ at any point in $\Omega_{+}$. Clearly then $\dot{U}=0$ at any point in $\Omega_{+}$and so by Eq. (16), $\dot{\varphi}=0$ at any point in $\Omega_{+}$. This means that $\varphi$ is constant in time and so by the initial condition (2) must also be zero. It has therefore been shown that the Burton-Miller-type integral equation with homogeneous boundary condition (12) does not admit nontrivial solutions. Recall from the previous section that the stability problems inherent in the Kirchoff integral equation are related to its admission of resonant time harmonic solutions at some time when the initial conditions have vanished and the boundary sources are controlling the problem. The above-presented argument shows that the Burton-Miller-type integral equation (10) does not allow any resonant solutions and hence does not suffer from these stability problems.

\section{THE SPACE-TIME DISCRETIZATION}

Equation (10) is discretized in space and time in order to obtain a numerical solution via the collocation method. To do this, $\Gamma$ is divided into $n$ elements and the time axis into a regular grid $t_{k}=(k-1) \Delta t, k=1, \ldots, N$, where $\Delta t$ is the time step. The solution $\varphi(\underline{x}, t)$ is represented in terms of basis functions $\psi_{j}(\underline{x}), j=1, \ldots, n$ which interpolate $\varphi$ in space, and $T_{k}(t), k=1, \cdots, N$ which interpolate $\varphi$ in time. Explicitly this yields

$$
\varphi(\underline{x}, t)=\sum_{k=1}^{N} \sum_{j=1}^{n} \varphi_{j}^{k} \psi_{j}(\underline{x}) T_{k}(t),
$$

where $\varphi_{j}^{k}$ are unknown coefficients. Piecewise constant functions are chosen for $\psi_{j}, j=1, \ldots, n$ as these simplify the evaluation of the hypersingular integral later on. Cubic time interpolation functions as used in Ref. 12 are chosen for $T_{k}$, $k=1, \ldots, N$ and are given by $T_{k}(t)=T\left(t-t_{k}\right)$, where

$T(t)$

$$
=\left\{\begin{array}{cc}
1+\frac{11}{6}\left(\frac{t}{\Delta t}\right)+\left(\frac{t}{\Delta t}\right)^{2}+\frac{1}{6}\left(\frac{t}{\Delta t}\right)^{3}, & -\Delta t<t \leqslant 0 \\
1+\frac{1}{2}\left(\frac{t}{\Delta t}\right)-\left(\frac{t}{\Delta t}\right)^{2}-\frac{1}{2}\left(\frac{t}{\Delta t}\right)^{3}, & 0<t \leqslant \Delta t \\
1-\frac{1}{2}\left(\frac{t}{\Delta t}\right)-\left(\frac{t}{\Delta t}\right)^{2}+\frac{1}{2}\left(\frac{t}{\Delta t}\right)^{3}, & \Delta t<t \leqslant 2 \Delta t \\
1-\frac{11}{6}\left(\frac{t}{\Delta t}\right)+\left(\frac{t}{\Delta t}\right)^{2}-\frac{1}{6}\left(\frac{t}{\Delta t}\right)^{3}, & 2 \Delta t<t \leqslant 3 \Delta t \\
0 & \text { otherwise. }
\end{array}\right.
$$

Figure 1 gives a plot of the nonzero part of $T(t)$ and this choice ensures that all quantities evaluated in Eq. (10) are interpolated by at least piecewise linear functions in time. 
PROOF COPY 010607JAS

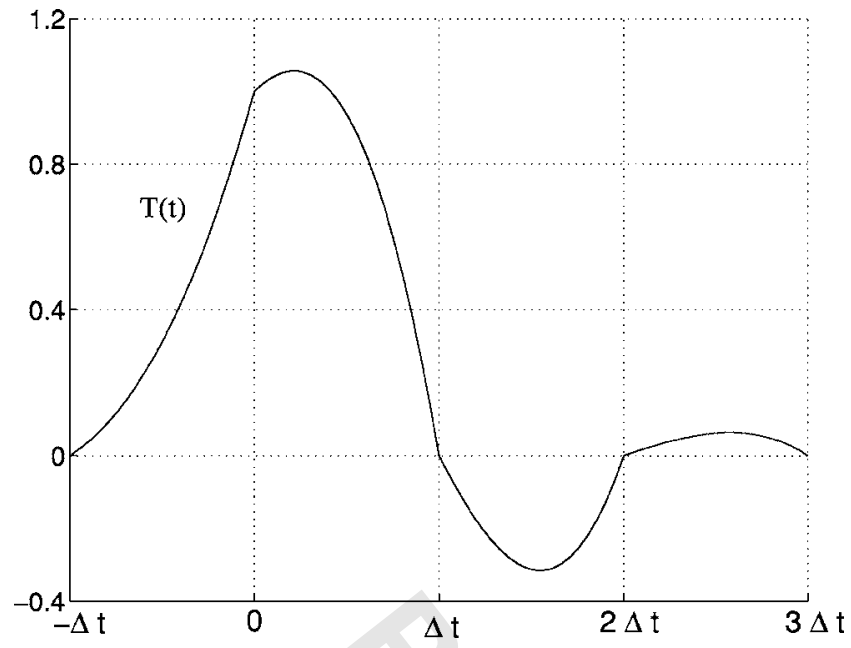

FIG. 1. The nonzero part of the cubic temporal basis function $T(t)$.

Expanding Eq. (10) out in the form of Eq. (6), substituting in Eq. (17) and evaluating at the $k$ th time step yields an equation of the form

$$
-\mathbf{A}^{(0)} \underline{\varphi}_{k}=\sum_{l=1}^{k-1} \mathbf{A}^{(l)} \underline{\varphi}_{k-1}+\underline{y}_{k}
$$

The terms in this matrix equation are given by

$$
\begin{aligned}
& A_{i, j}^{(l)}=(1-\alpha)\left\{\frac{-1}{4 \pi} \int_{\Gamma_{j}} \frac{\partial R}{\partial \hat{n}_{\xi}}\left[\frac{\dot{T}_{k-l}\left(\tau_{k}\right)}{R^{2}}+\frac{\ddot{T}_{k-l}\left(\tau_{k}\right)}{c R}\right] d S_{\xi}\right. \\
& \left.-\frac{1}{2} \delta_{i, j} \dot{T}_{k-l}\left(t_{k}\right)\right\}+\frac{\alpha c}{4 \pi}\left\{f _ { \Gamma _ { j } } \frac { \partial ^ { 2 } } { \partial \underline { \hat { n } } _ { x } \partial \underline { \hat { n } } _ { \xi } } ( \frac { 1 } { R } ) \left[T_{k-l}\left(\tau_{k}\right)\right.\right. \\
& \left.\left.+\frac{R}{c} \dot{T}_{k-l}\left(\tau_{k}\right)\right] d S_{\xi}+\int_{\Gamma_{j}} \frac{1}{c^{2} R} \frac{\partial R}{\partial \underline{\hat{n}}_{x}} \frac{\partial R}{\partial \underline{\hat{n}}_{\xi}} \ddot{T}_{k-l}\left(\tau_{k}\right) d S_{\xi}\right\}, \\
& y_{k}=\frac{-(1-\alpha)}{4 \pi} \int_{\Gamma} \frac{1}{R}\left(\frac{\dot{\partial} \varphi}{\partial \hat{n}_{\xi}}\right)\left(\underline{\xi}, \tau_{k}\right) d S_{\xi} \\
& +\alpha c\left\{\frac { 1 } { 4 \pi } \int _ { \Gamma } \frac { \partial R } { \partial \underline { \hat { n } } _ { x } } \left[\frac{1}{R^{2}} \frac{\partial \varphi}{\partial \hat{n}_{\xi}}\left(\underline{\xi}, \tau_{k}\right)\right.\right. \\
& \left.\left.+\frac{1}{c R}\left(\frac{\dot{\partial} \varphi}{\partial \hat{n}_{\xi}}\right)\left(\underline{\xi}, \tau_{k}\right)\right] d S_{\xi}-\frac{1}{2} \frac{\partial \varphi}{\partial \underline{n}_{x}}\left(\underline{x}, t_{k}\right)\right\}, \\
& \varphi_{k}=\left[\varphi_{1}^{k}, \ldots, \varphi_{n}^{k}\right]^{T},
\end{aligned}
$$

where $f$ denotes the Hadamard finite part integral, $R=\mid \underline{x}_{i}$ $-\underline{\xi} \mid, \tau_{k}=t_{k}-R / c$ and $\delta_{i, j}$ is the Kronecker delta.

The surface solution at any given time $t_{k}$ may be generated using Eq. (19), by starting at the first time step and solving recursively (time marching) until reaching the desired time. The exterior solution at some point $\underline{x} \in \Omega_{+}$may then be calculated using the discrete form of Eq. (6). The finite part integral is used since the integral here is hypersingular and so a method must be developed to evaluate its finite part numerically.

\section{A. Evaluating the hypersingular integral}

The hypersingular integral occurring in Eq. (10) can be seen in its discretized form as the Hadamard finite part integral appearing in terms of the matrix $\mathbf{A}$ in the previous section. Before discretization, this integral was of the form

with notation as in Sec. II. As $R \rightarrow 0$, the first term in Eq. (21) is $O\left(R^{-3}\right)$ and the second is $O\left(R^{-2}\right)$. This can be reduced to the weakly singular case $\left(O\left(R^{-1}\right)\right)$ by applying Taylor's theorem as follows:

$$
\begin{aligned}
& \varphi(\underline{\xi}, \tau)=\varphi(\underline{x}, t)+\left.(\underline{\xi}-\underline{x}) \cdot \nabla_{\xi}[\varphi(\underline{\xi}, \tau)]\right|_{\xi=x}+O\left(R^{2}\right), \\
& \dot{\varphi}(\underline{\xi}, \tau)=\dot{\varphi}(\underline{x}, t)+O(R),
\end{aligned}
$$

which is valid if $\dot{\varphi}$ is differentiable in space on $\Gamma \times[0, \infty)$ and $\varphi$ is twice differentiable in space on $\Gamma \times[0, \infty)$. Applying this to the hypersingular integral the following expression which is equivalent to Eq. (21) is obtained

$$
\begin{aligned}
& \int_{\Gamma} \frac{\partial^{2}}{\partial \underline{\hat{n}}_{x} \partial \underline{\hat{n}}_{\xi}}\left(\frac{1}{R}\right)[\varphi(\underline{\xi}, \tau)-\varphi(\underline{x}, t)-(\underline{\xi}-\underline{x}) \\
& \left.\left.\cdot \nabla_{\xi}[\varphi(\underline{\xi}, \tau)]\right|_{\xi=x}\right] d S_{\xi}+\int_{\Gamma} \frac{R}{c} \frac{\partial^{2}}{\partial \underline{\hat{n}}_{x} \partial \underline{\hat{n}}_{\xi}}\left(\frac{1}{R}\right)[\dot{\varphi}(\underline{\xi}, \tau) \\
& -\dot{\varphi}(\underline{x}, t)] d S_{\xi}+\varphi(\underline{x}, t) \int_{\Gamma} \frac{\partial^{2}}{\partial \hat{\hat{n}}_{x} \partial \underline{\hat{n}}_{\xi}}\left(\frac{1}{R}\right) d S_{\xi} \\
& +\left.f_{\Gamma} \frac{\partial^{2}}{\partial \underline{\hat{n}}_{x} \partial \underline{\hat{n}}_{\xi}}\left(\frac{1}{R}\right)(\underline{\xi}-x) \cdot \nabla_{\xi}[\varphi(\underline{\xi}, \tau)]\right|_{\xi=x} d S_{\xi} \\
& +\dot{\varphi}(\underline{x}, t) f_{\Gamma} \frac{R}{c} \frac{\partial^{2}}{\partial \underline{\hat{n}}_{x} \partial \underline{\hat{n}}_{\xi}}\left(\frac{1}{R}\right) d S_{\xi} .
\end{aligned}
$$

This can now be evaluated since the first two integrals are weakly singular, the third may be evaluated using an identity from Meyer et al. ${ }^{15}$ and it is easy to show that the last two integrals cancel in the case of piecewise constant spatial basis functions. The identity from Meyer gives that

$$
f_{\Gamma} \frac{\partial^{2}}{\partial \underline{\hat{n}}_{x} \partial \underline{\hat{n}}_{\xi}}\left(\frac{e^{i k R}}{R}\right) d S_{\xi}=k^{2} \int_{\Gamma}\left(\underline{\hat{n}}_{x} \cdot \underline{\hat{n}}_{\xi}\right) \frac{e^{i k R}}{R} d S_{\xi},
$$

and so setting $k=0$, this can be used to evaluate the third integral in Eq. (23). A method for evaluating the hypersingular integral which is valid for any type of surface approximation has therefore been developed.

This is important for applications such as loudspeakers where many elements would be required for flat elements to provide an accurate discretization.

\section{NUMERICAL RESULTS}

The examples studied in this work are restricted to radiation from axisymmetric objects as this, simplifies the calculations. Figure 1 shows the generating curves for the three objects considered. The generating curve for the peanut is defined by three unit circles whose centers lie on an equilateral triangle. Starting from the point $(0,2)$ at the top of the curve and moving along it in a clockwise direction, the first 

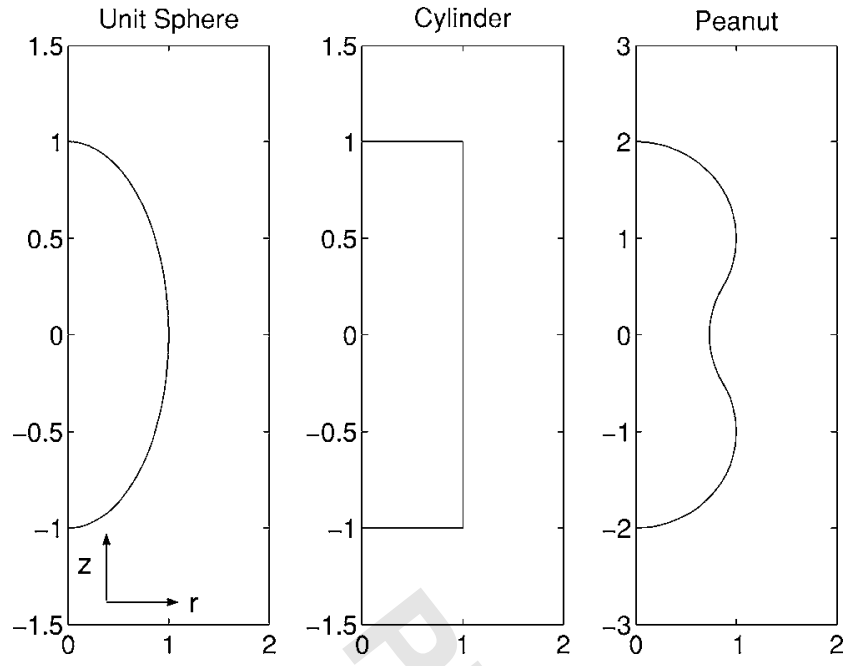

FIG. 2. Generating curves for the axisymmetric radiating objects considered.

$2 / 5$ of the arclength is formed by an arc from a circle centered at $(0,1)$, the next $1 / 5$ from a circle centered at $(\sqrt{3}, 0)$ and the final $2 / 5$ from a circle centered at $(0,-1)$. All meshes used to approximate $\Gamma$ are exact geometric representations defined in terms of arcs of circles or straight lines as appropriate.

In all cases the radiation of a spherically symmetric wave defined by

$$
\begin{aligned}
\varphi(R, t)= & \frac{1}{R}\left(\frac{3}{4}-\cos \left(\frac{\pi(R-c t+3 a)}{2 a}\right)\right. \\
& \left.+\frac{1}{4} \cos \left(\frac{\pi(R-c t+3 a)}{a}\right)\right)(H(R-c t+3 a) \\
& -H(R-c t-a))
\end{aligned}
$$

is considered, which has the property that $(\dot{\partial} \varphi / \partial \hat{n})$ is continuous in time. Here $a$ is the radius of some sphere $S \subseteq \Omega, R$ is the distance from the center of $\mathrm{S}$ to some point $\underline{x} \in \Omega_{+}$, and $H$ is the Heaviside step function. It may be verified that Eq. (25) satisfies Eqs. (1)-(3) from the initial-boundary value problem. The boundary data required in Eq. (4) may be calculated from Eq. (25) using the chain rule and simplifications due to the axisymmetric geometry to give

$$
\frac{\partial \varphi}{\partial r}=\frac{\partial \varphi}{\partial R} \sin \theta, \quad \frac{\partial \varphi}{\partial z}=\frac{\partial \varphi}{\partial R} \cos \theta,
$$

where $r$ and $z$ are the coordinate axes shown in Fig. 2 and $\theta$ is the angle measured clockwise from the positive $z$ axis. Hence the boundary data are given by

$$
\frac{\partial \varphi}{\partial \underline{\hat{n}}}=n_{r} \frac{\partial \varphi}{\partial r}+n_{z} \frac{\partial \varphi}{\partial z}=\frac{\partial \varphi}{\partial R}\left[n_{r} \sin \theta+n_{z} \cos \theta\right],
$$

where $n_{r}$ and $n_{z}$ are the components of $\hat{n}$ in the $r$ and $z$ directions, respectively. It is also straightforward to compute $(\dot{\partial} \varphi / \partial \hat{n})$ from the above. In the results that follow $\mathrm{S}$ is taken to be the sphere centered at the origin with the maximum possible radius $a$ such that $\mathrm{S} \subseteq \Omega$ and the speed of sound has been normalized so that $c=1$. A Fourier transform shows that
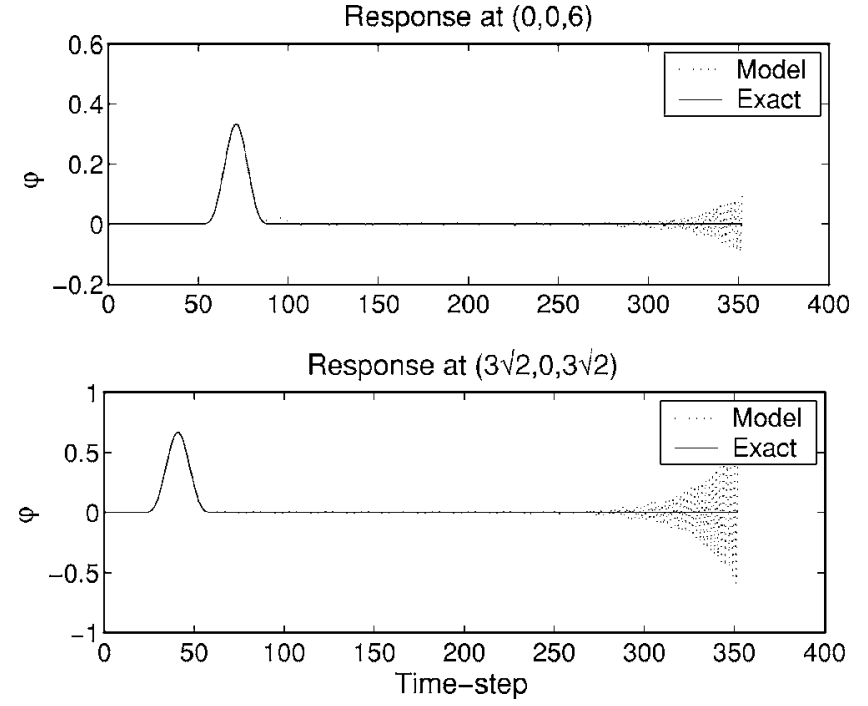

FIG. 3. The velocity potential calculated at two exterior points using the Kirchoff equation to calculate the surface solution on a unit sphere. Results are compared with an exact solution.

the wave defined by Eq. (25) is essentially band limited at frequency $f_{\max }$, which depends on the value of $a$. For accuracy, the time step is chosen as $\Delta t=1 /\left(10 f_{\max }\right)$ and the coupling parameter $\alpha$ is taken to be 0.5 as in Ref. 12 .

The first example to be considered is that of a unit sphere. Since the radiated waves are spherically symmetric, the surface solution will be constant over $\Gamma$ and so piecewise constant spatial basis functions will provide a good approximation of the analytic solution. Hence the relatively small value of $n=10$ boundary elements are used. The time step is taken to be $1 / 10$ for the above-given reasons. This test problem is first solved using the simpler Kirchoff integral equation (6). Here the more widely used linear hat functions are applied for the time interpolation as in Ref. 5. Figure 3 shows the numerical results for $\varphi$ compared with the exact solution at two different points in the exterior field. The results are clearly unstable, increasing exponentially after around 250 time steps. The results of repeating the same test problem, but using the Burton-Miller-type integral equation (10) are shown in Fig. 4. Here the results match the exact solution closely and so plots of the relative error at the two exterior points are given instead of the numerical and exact solutions. The relative error is Calculated from ( $\varphi$ $-\widetilde{\varphi}) / \max \varphi$, where $\varphi$ and $\widetilde{\varphi}$ denote the exact and numerical solutions, respectively. Note that the "max" used in the denominator is taken with respect to time and is used to avoid division by zero. The dotted vertical lines on the error plots in this paper are used to indicate the position of the radiated pulse shown in Fig. 3. The error is very small, peaking at 0.002783 for the point $(0,0,6)$ and at 0.003293 for the point $(3 \sqrt{2}, 0,3 \sqrt{2})$. These peaks both occurred during the pulse. This shows that the results are accurate and stable over the 352 time steps shown.

Now the long-term stability of the method for calculating the surface solution is demonstrated. This is shown in terms of the characteristic ratio $c T / \operatorname{diam}(\Omega)$ as in Ref. 10, where $T$ is the maximum time at which the solution is evaluated and $\operatorname{diam}(\Omega)$ is the diameter of $\Omega$. In Ref. 10 long-term 

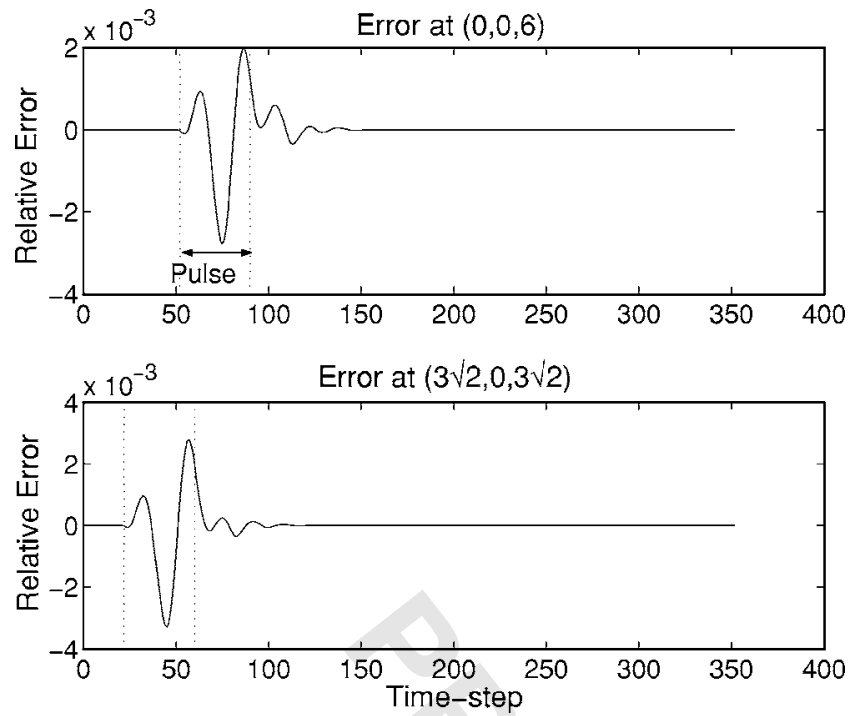

FIG. 4. The time variation of the relative error calculated at two exterior points using the Burton-Miller-type equation to calculate the surface solution on a unit sphere.

stability was shown with a characteristic ratio of greater than 500. Figure 5 shows the surface solution at the ten surface collocation points with a characteristic ratio of greater than 1000. This clearly shows the stability of the results over a long period of time (2002 s) and surpasses previous results.

Now the other examples of a cylinder and a peanut shaped mesh are considered. Here the spherical wave is not constant over $\Gamma$ and so finer spatial discretizations are required for accurate results. More elements are also required since these objects have longer arclengths than the sphere. For the cylinder, $n=40$ is used and $\Delta t=1 / 10$ for the abovenoted reasons. Figure 6 shows how the relative error varies in time at the same two points in the exterior field as before. The error is fairly small, peaking at 0.007157 after the pulse for the point $(0,0,6)$ and at 0.01182 during the pulse for the point $(3 \sqrt{2}, 0,3 \sqrt{2})$. These peaks are greater than for the sphere, but this is not surprising given that the cylinder is not a regular surface as it has edges which are well known to have a detrimental effect on the accuracy of boundary ele-

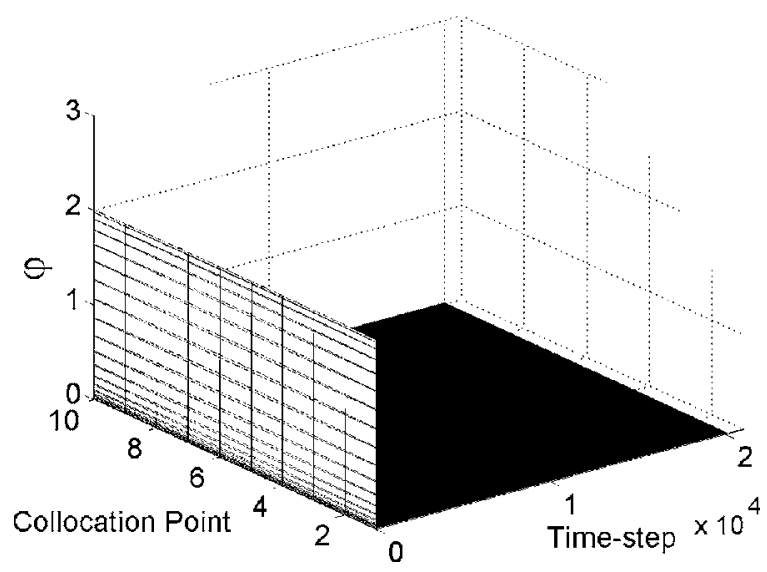

FIG. 5. The velocity potential calculated at the surface collocation points of the unit sphere, showing stability of the Burton-Miller-type equation for 20020 time steps.
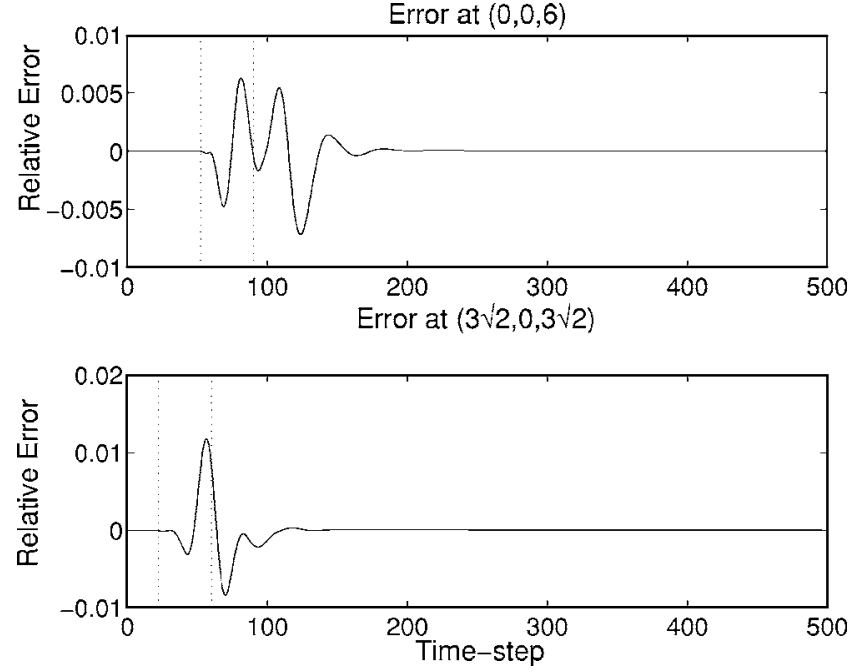

FIG. 6. The time variation of the relative error calculated at two exterior points using the Burton-Miller-type equation to calculate the surface solution on a cylinder.

ment methods. However, the results are still reasonably accurate and stable over the 496 time steps shown.

For the peanut, $f_{\max }$ is larger than in the previous examples and so a smaller time step of $1 / 14$ is used. This together with the fact that the peanut shaped mesh has the longest arclength of the examples considered means that the relatively large value of $n=60$ is taken. Figure 7 shows the results for the relative error in the exterior field, again at the same two points. The error is very small, peaking at 0.005428 for the point $(0,0,6)$ and at 0.003742 for the point $(3 \sqrt{2}, 0,3 \sqrt{2})$. These peaks both occurred just before the end of the pulse. The errors shown in this case are smaller than for the cylinder but larger than for the sphere. This is the expected result since the peanut is a regular surface but the radiated wave is not constant over $\Gamma$ like it is for the sphere. Therefore the results are accurate and stable over the 928 time steps shown.
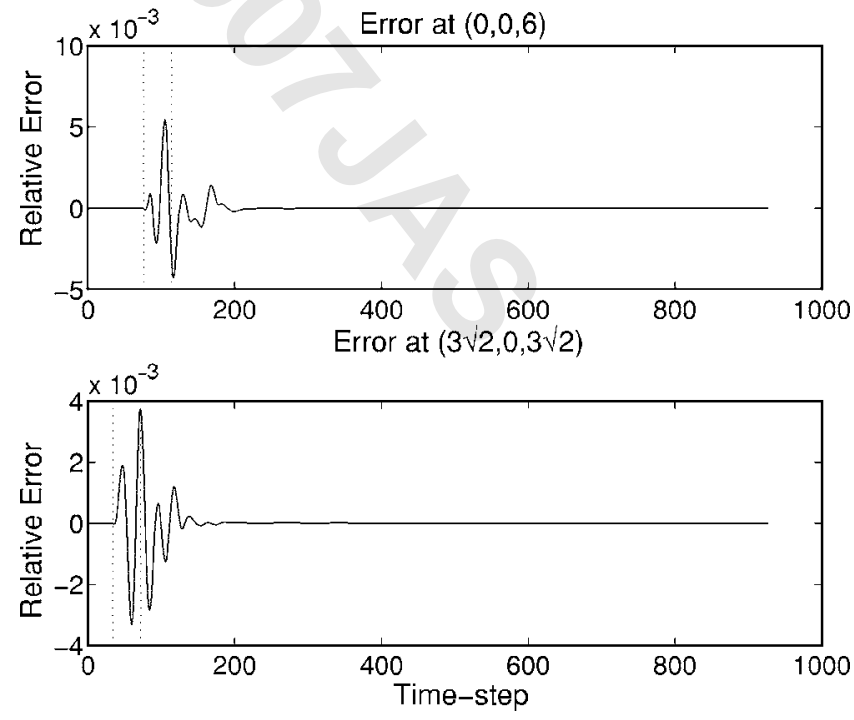

FIG. 7. The time variation of the relative error calculated at two exterior points using the Burton-Miller-type equation to calculate the surface solution on a peanut shaped axisymmetric object. 
PROOF COPY 010607JAS

\section{CONCLUSIONS}

The methods presented accurately modeled transient acoustic radiation from various geometric objects. Furthermore, the Burton-Miller-type integral equation formulation employed was shown to be free of the long-time instabilities often suffered by such methods. The numerical results shown support these observations and a comparison with the more commonly used (but unstable) Kirchoff formulation was given. The hypersingular integrals arising in the BurtonMiller-type integral equation are reformulated as weakly singular ones using piecewise constant collocation and may be calculated for any choice of surface discretization.

\section{ACKNOWLEDGMENT}

The work carried out in this paper was partially funded by B\&W Group Limited, Worthing, West Sussex, UK.

${ }^{1}$ M. B. Friedman and R. Shaw, "Diffraction of pulses by cylindrical objects of arbitrary cross section," J. Appl. Mech. 29, 40-46 (1962).

${ }^{2}$ K. M. Mitzner, "Numerical solution for transient scattering from a hard surface of arbitrary shape-retarded potential technique," J. Acoust. Soc. Am. 42, 391-397 (1967).

${ }^{3}$ B. P. Rynne, "Stability and convergence of time marching methods in scattering problems," IMA J. Appl. Math. 35, 297-310 (1985).

${ }^{4}$ B. Birgisson, E. Siebrits, and A. P. Pierce, "Elastodynamic direct boundary element methods with enhanced numerical stability properties," Int. J. Numer. Methods Eng. 46, 871-888 (1999).

${ }^{5} \mathrm{H}$. Wang, "Boundary integral modelling of transient wave propagation with application to acoustic radiation from loudspeaker," Ph.D. thesis, University of Brighton, 2004.
${ }^{6}$ B. P. Rynne and P. D. Smith, "Stability of time marching algorithms for the electric field integral equation," J. Electromagn. Waves Appl. 4(12), 1181-1205 (1990).

${ }^{7}$ P. D. Smith, "Instabilities in time marching methods for scattering: Cause and rectification," Electromagnetics 10, 439-451 (1990).

${ }^{8}$ M. J. Bluck and S. P. Walker, "Analysis of three-dimensional transient acoustic wave propagation using the boundary integral equation method," Int. J. Numer. Methods Eng. 39, 1419-1431 (1996).

${ }^{9}$ S. J. Dodson, S. P. Walker, and M. J. Bluck, "Implicitness and stability of time domain integral equation scattering analysis," Appl. Comput. Electromagn. Soc. J. 13(3), 291-301 (1998).

${ }^{10}$ T. Ha-Duong, B. Ludwig, and I. Terrasse, "A Galerkin BEM for transient acoustic scattering by an absorbing obstacle,” Int. J. Numer. Methods Eng. 57, 1845-1882 (2003).

${ }^{11}$ A. J. Burton and G. F. Miller, "The application of integral equation methods to the numerical solution of some exterior boundary-value problems," Proc. R. Soc. London, Ser. A 323, 201-210 (1971).

${ }^{12}$ A. A. Ergin, B. Shanker, and E. Michielssen, "Analysis of transient wave scattering from rigid bodies using a Burton-Miller approach," J. Acoust. Soc. Am. 106(5), 2396-2404 (1999).

${ }^{13} \mathrm{~T}$. Terai, "On the calculation of sound fields around three dimensional objects by integral equation methods," J. Sound Vib. 69(1), 71-100 (1980).

${ }^{14}$ Y. Kawai and T. Terai, "A numerical method for the calculation of transient acoustic scattering from thin rigid plates," J. Sound Vib. 141( 1), 83-96 (1990).

${ }^{15}$ W. L. Meyer, W. A. Bell, and B. T. Zinn, "Boundary integral solutions of three dimensional acoustic radiation problems," J. Sound Vib. 59(2), 245262 (1978).

${ }^{16} \mathrm{M}$. Costabel, "Time-dependent problems with the boundary integral equation method," Encyclopedia of Comp. Mechanics, edited by E. Stein, R. de Borst, and T. Hughes (Wiley, New York, 2004), Chap. 22.

${ }^{17}$ C. A. Coulson and A. Jeffrey, Waves: A Mathematical Approach to the Common Types of Wave Motion (Longman Group, London, 1977). 